\title{
On the identity involving certain Hardy sums and Kloosterman sums
}

Han Zhang ${ }^{*}$ and Wenpeng Zhang

"Correspondence:

micohanzhang@gmail.com

Department of Mathematics,

Northwest University, Xi'an, Shaanxi, P.R. China

\section{Abstract \\ The main purpose of this paper is, using the properties of Gauss sums and the mean value theorem of Dirichlet $L$-functions, to study a hybrid mean value problem involving certain Hardy sums and Kloosterman sums and give two exact computational formulae for them.}

MSC: 11L05; 11M20

Keywords: Gauss sums; Kloosterman sums; identity; certain Hardy sums; hybrid mean value; computational formula

\section{Introduction}

Let $c$ be a natural number and $d$ be an integer prime to $c$. The classical Dedekind sums

$$
S(d, c)=\sum_{j=1}^{c}\left(\left(\frac{j}{c}\right)\right)\left(\left(\frac{d j}{c}\right)\right),
$$

where

$$
((x))= \begin{cases}x-[x]-\frac{1}{2} & \text { if } x \text { is not an integer } \\ 0 & \text { if } x \text { is an integer }\end{cases}
$$

describes the behavior of the logarithm of the eta-function (see [1] and [2]) under modular transformations. Berndt [3] gave an analogous transformation formula for the logarithm of the classical theta-function

$$
\theta(z)=\sum_{n=-\infty}^{+\infty} \exp \left(\pi i n^{2} z\right), \quad \operatorname{Im}(z)>0
$$

That is, put $V z=(a z+b)(c z+d)$ with $a, b, c, d \in Z, c>0$, and $a d-b c=1$. Then we have

$$
\log \theta(V z)=\log \theta(z)+\frac{1}{2} \log (c z+d)-\frac{1}{4} \pi i+\frac{1}{4} \pi i S_{1}(d, c)
$$

where $S_{1}(d, c)$ are defined as

$$
S_{1}(d, c)=\sum_{j=1}^{c-1}(-1)^{j+1+\left[\frac{d j}{c}\right]}
$$


The sums $S_{1}(d, c)$ (and certain related ones) are sometimes called Hardy sums. They are closely connected with Dedekind sums. Some authors had studied the properties of $S_{1}(d, c)$ and related sums and obtained some interesting results, see [4-8] and [9]. For example, Zhang and Yi [8] proved the following conclusion. Let $p$ be an odd prime. Then, for any fixed positive integer $m$, we have the asymptotic formula

$$
\sum_{h=1}^{p-1}\left|S_{1}(h, p)\right|^{2 m}=p^{2 m} \cdot \frac{\zeta^{2}(2 m)\left(1-\frac{1}{4^{m}}\right)}{\zeta(4 m)\left(1+\frac{1}{4^{m}}\right)}+O\left(p^{2 m-1} \cdot \exp \left(\frac{6 \ln p}{\ln \ln p}\right)\right),
$$

where $\zeta(s)$ is the Riemann zeta-function and $\exp (y)=e^{y}$.

On the other hand, we introduce the classical Kloosterman sums $K(n, q)$ which are defined as follows: For any positive integer $q>1$ and integer $n$,

$$
K(n, q)=\sum_{b=1}^{q} e\left(\frac{n b+\bar{b}}{q}\right)
$$

where $\bar{b}$ denotes the solution of the congruence $x \cdot b \equiv 1 \bmod q, \sum_{b=1}^{\prime q}$ denotes the summation over all $1 \leq b \leq q$ with $(b, q)=1$ and $e(x)=e^{2 \pi i x}$. Some elementary properties of $K(n, q)$ can be found in [10] and [11].

The main purpose of this paper is, using the properties of Gauss sums and the mean square value theorem of Dirichlet $L$-functions, to study a hybrid mean value problem involving certain Hardy sums and Kloosterman sums and give two exact computational formulae. That is, we shall prove the following theorem.

Theorem 1 Let $p$ be an odd prime. Then we have the identity

$$
\sum_{m=1}^{p-1} \sum_{n=1}^{p-1} K(m, p) \cdot K(n, p) \cdot S_{1}(2 m \cdot \bar{n}, p)= \begin{cases}2 p^{2} & \text { if } p \equiv 3 \bmod 4 \\ 0 & \text { if } p \equiv 1 \bmod 4\end{cases}
$$

Theorem 2 Let $p$ be an odd prime, then we have the identity

$$
\begin{aligned}
& \sum_{m=1}^{p-1} \sum_{n=1}^{p-1}|K(m, p)|^{2} \cdot|K(n, p)|^{2} \cdot S_{1}(2 m \cdot \bar{n}, p) \\
& \quad= \begin{cases}2 p^{3}+4 \cdot p^{2} \cdot h_{p}^{2} & \text { if } p \equiv 7 \bmod 8 \\
2 p^{3}-36 \cdot p^{2} \cdot h_{p}^{2} & \text { if } p \equiv 3 \bmod 8 \\
0 & \text { if } p \equiv 1 \bmod 4\end{cases}
\end{aligned}
$$

where $h_{p}$ denotes the class number of the quadratic field $\mathbf{Q}(\sqrt{-p})$.

For general odd number $q \geq 3$, whether there exits a computational formula for the hybrid mean value

$$
\sum_{m=1}^{q} \sum_{n=1}^{q}|K(m, q)|^{2} \cdot|K(n, q)|^{2} \cdot S_{1}(2 m \cdot \bar{n}, q)
$$

is an open problem. 


\section{Several lemmas}

In this section, we shall give several lemmas, which are necessary in the proof of our theorems. Hereinafter, we shall use many properties of Gauss sums, all of which can be found in [12], so they will not be repeated here. First we have the following lemma.

Lemma 1 Let $p$ be an odd prime, then we have the identity

$$
\sum_{n=1}^{p-1} \chi(n) \cdot|K(n, p)|^{2}=\bar{\chi}(-1) \cdot \frac{\tau^{3}(\chi) \cdot \tau\left(\bar{\chi}^{2}\right)}{\tau(\bar{\chi})} .
$$

Proof For any non-principal character $\chi \bmod p$, from the properties of Gauss sums we have

$$
\begin{aligned}
\sum_{n=1}^{p-1} \chi(n)|K(n, p)|^{2} & =\sum_{a=1}^{p-1} \sum_{b=1}^{p-1} \sum_{n=1}^{p-1} \chi(n) e\left(\frac{n(a-b)+(\bar{a}-\bar{b})}{p}\right) \\
& =\sum_{a=1}^{p-1} \sum_{b=1}^{p-1} \sum_{n=1}^{p-1} \chi(n) e\left(\frac{n b(a-1)+\bar{b}(\bar{a}-1)}{p}\right) \\
& =\tau(\chi) \cdot \sum_{a=1}^{p-1} \sum_{b=1}^{p-1} \bar{\chi}(b(a-1)) e\left(\frac{\bar{b}(\bar{a}-1)}{p}\right) \\
& =\tau^{2}(\chi) \cdot \sum_{a=1}^{p-1} \bar{\chi}(a-1) \bar{\chi}(\bar{a}-1) \\
& =\tau^{2}(\chi) \cdot \sum_{a=1}^{p-1} \chi(a) \bar{\chi}\left(-(a-1)^{2}\right)=\bar{\chi}(-1) \cdot \tau^{2}(\chi) \cdot \sum_{a=1}^{p-2} \chi(a+1) \bar{\chi}\left(a^{2}\right) \\
& =\bar{\chi}(-1) \cdot \tau^{2}(\chi) \cdot \sum_{a=1}^{p-2} \chi\left(\bar{a}+\bar{a}^{2}\right)=\bar{\chi}(-1) \cdot \tau^{2}(\chi) \cdot \sum_{a=1}^{p-1} \chi\left(a^{2}+a\right) \\
& =\bar{\chi}(-1) \cdot \tau^{2}(\chi) \cdot \frac{1}{\tau(\bar{\chi})} \sum_{a=1}^{p-1} \sum_{b=1}^{p-1} \bar{\chi}(b) e\left(\frac{b\left(a^{2}+a\right)}{p}\right) \\
& =\bar{\chi}(-1) \cdot \tau^{2}(\chi) \cdot \frac{1}{\tau(\bar{\chi})} \cdot \sum_{b=1}^{p-1} \bar{\chi}(b) e\left(\frac{b}{p}\right) \sum_{a=1}^{p-1} \chi(a) e\left(\frac{b a}{p}\right) \\
& =\bar{\chi}(-1) \cdot \tau^{3}(\chi) \cdot \frac{1}{\tau(\bar{\chi})} \cdot \sum_{b=1}^{p-1} \bar{\chi}^{2}(b) e\left(\frac{b}{p}\right) \\
\tau(\bar{\chi}) & \tau^{3}(\chi) \cdot \tau\left(\bar{\chi}^{2}\right) \\
& \\
& \\
&
\end{aligned}
$$

This proves Lemma 1.

Lemma 2 Let $q>2$ be an integer, then, for any integer a with $(a, q)=1$, we have the identity

$$
S(a, q)=\frac{1}{\pi^{2} q} \sum_{d \mid q} \frac{d^{2}}{\phi(d)} \sum_{\substack{\chi \bmod d \\ \chi(-1)=-1}} \chi(a)|L(1, \chi)|^{2},
$$

where $L(1, \chi)$ denotes the Dirichlet L-function corresponding to character $\chi$ mod $d$. 
Proof See Lemma 2 of [9].

Lemma 3 Let $q>0$ and $(h, q)=1$. Then we have the identity

$$
S_{1}(h, q)=-8 S(h+q, 2 q)+4 S(h, q) \text {. }
$$

Proof This formula is an immediate consequence of (5.9) and (5.10) in [7].

Lemma 4 Let $p$ be an odd prime and $0<h<p$. Then we have the identity

$$
S_{1}(2 h, p)=-20 \cdot S(2 h, p)+8 \cdot S(4 h, p)+8 \cdot S(h, p) .
$$

Proof Note that the divisors of $2 p$ are 1, 2, $p$ and $2 p$. So from Lemma 2 and Lemma 3 we have

$$
\begin{aligned}
S_{1}(2 h, p)= & -8 \cdot S(2 h+p, 2 p)+4 \cdot S(2 h, p) \\
= & -\frac{4}{\pi^{2} p} \sum_{d \mid 2 p} \frac{d^{2}}{\phi(d)} \sum_{\substack{\chi \bmod d \\
\chi(-1)=-1}} \chi(2 h+p)|L(1, \chi)|^{2} \\
& +\frac{4}{\pi^{2} p} \sum_{d \mid p} \frac{d^{2}}{\phi(d)} \sum_{\chi \bmod d} \chi(2 h)|L(1, \chi)|^{2} \\
= & -\frac{4}{\pi^{2} p} \cdot \frac{(2 p)^{2}}{\phi(2 p)} \sum_{\substack{\chi \bmod _{2} p \\
\chi(-1)=-1}} \chi(2 h+p)|L(1, \chi)|^{2} \\
= & -\frac{16 p}{\pi^{2}(p-1)} \cdot \sum_{\substack{\chi \bmod p \\
\chi(-1)=-1}} \chi(2 h+p) \lambda(2 h+p)|L(1, \chi \lambda)|^{2} \\
= & -\frac{16 p}{\pi^{2}(p-1)} \cdot \sum_{\substack{\chi \bmod p \\
\chi(-1)=-1}} \chi(2 h)|L(1, \chi \lambda)|^{2},
\end{aligned}
$$

where $\lambda$ denotes the principal character mod 2 .

From the Euler infinite product formula we have

$$
\begin{aligned}
|L(1, \chi \lambda)|^{2} & =\prod_{p_{1}}\left|1-\frac{\chi\left(p_{1}\right) \lambda\left(p_{1}\right)}{p_{1}}\right|^{-2}=\prod_{p_{1}>2}\left|1-\frac{\chi\left(p_{1}\right)}{p_{1}}\right|^{-2} \\
& =\left|1-\frac{\chi(2)}{2}\right|^{2} \cdot \prod_{p_{1}}\left|1-\frac{\chi\left(p_{1}\right)}{p_{1}}\right|^{-2}=\left(\frac{5}{4}-\frac{\chi(2)}{2}-\frac{\bar{\chi}(2)}{2}\right) \cdot|L(1, \chi)|^{2},
\end{aligned}
$$

where $\prod_{p}$ denotes the product over all primes $p$.

From Lemma 2 we also have the identity

$$
S(n, p)=\frac{1}{\pi^{2}} \cdot \frac{p}{p-1} \sum_{\substack{\chi \bmod p \\ \chi(-1)=-1}} \chi(n)|L(1, \chi)|^{2} .
$$


Now, combining (2), (3) and (4), we have the identity

$$
\begin{aligned}
S_{1}(2 h, p) & =-\frac{16 p}{\pi^{2}(p-1)} \cdot \sum_{\substack{\chi \bmod p \\
\chi(-1)=-1}} \chi(2 h)|L(1, \chi \lambda)|^{2} \\
& =-16 \cdot \frac{p}{\pi^{2}(p-1)} \cdot \sum_{\substack{\chi \bmod p \\
\chi(-1)=-1}} \chi(2 h)\left(\frac{5}{4}-\frac{\chi(2)}{2}-\frac{\bar{\chi}(2)}{2}\right) \cdot|L(1, \chi)|^{2} \\
& =-20 \cdot S(2 h, p)+8 \cdot S(4 h, p)+8 \cdot S(h, p) .
\end{aligned}
$$

This proves Lemma 4.

Lemma 5 Let $p$ be an odd prime. Then we have the identities
(A) $\sum_{\substack{\chi \bmod p \\ \chi(-1)=-1}}|L(1, \chi)|^{2}=\frac{\pi^{2}}{12} \cdot \frac{(p-1)^{2} \cdot(p-2)}{p^{2}}$;
(B) $\sum_{\substack{\chi \bmod p \\ \chi(-1)=-1}} \chi(2) \cdot|L(1, \chi)|^{2}=\frac{\pi^{2}}{24} \cdot \frac{(p-1)^{2} \cdot(p-5)}{p^{2}}$;
(C) $\sum_{\substack{\chi \bmod p \\ \chi(-1)=-1}} \chi(4) \cdot|L(1, \chi)|^{2}= \begin{cases}\frac{\pi^{2}}{48} \cdot \frac{(p-1)^{2} \cdot(p-17)}{p^{2}} & \text { if } p \equiv 1 \bmod 4 ; \\ \frac{\pi^{2}}{48} \cdot \frac{(p-1) \cdot\left(p^{2}-6 p+17\right)}{p^{2}} & \text { if } p \equiv 3 \bmod 4 .\end{cases}$

Proof From the definition of Dedekind sums we have

$$
S(1, c)=\sum_{a=1}^{c-1}\left(\frac{a}{c}-\frac{1}{2}\right)^{2}=\frac{(c-1)(c-2)}{12 c} .
$$

If $p \equiv 1 \bmod c$, then from (5), and noting the reciprocity theorem of Dedekind sums (see [5]), we have the computational formula

$$
\begin{aligned}
S(c, p) & =\frac{p^{2}+c^{2}+1}{12 p c}-\frac{1}{4}-S(p, c)=\frac{p^{2}+c^{2}+1}{12 p c}-\frac{1}{4}-S(1, c) \\
& =\frac{p^{2}+c^{2}+1}{12 p c}-\frac{1}{4}-\frac{(c-1)(c-2)}{12 c}=\frac{(p-1)\left(p-1-c^{2}\right)}{12 p c} .
\end{aligned}
$$

If $p \equiv 3 \bmod 4$, then we also have

$$
\begin{aligned}
S(4, p) & =\frac{p^{2}+16+1}{48 p}-\frac{1}{4}-S(p, 4)=\frac{p^{2}+17}{48 p}-\frac{1}{4}-S(3,4) \\
& =\frac{p^{2}+17}{48 p}-\frac{1}{4}+\frac{1}{8}=\frac{p^{2}-6 p+17}{48 p} .
\end{aligned}
$$

Now taking $c=1$ in (6), from (4) we may immediately deduce the identity

$$
\sum_{\substack{\chi \bmod p \\ \chi(-1)=-1}}|L(1, \chi)|^{2}=\frac{\pi^{2}}{12} \cdot \frac{(p-1)^{2} \cdot(p-2)}{p^{2}} .
$$


Taking $c=2$ in (6), from (4) we can also deduce the identity

$$
\sum_{\substack{\chi \bmod p \\ \chi(-1)=-1}} \chi(2) \cdot|L(1, \chi)|^{2}=\frac{\pi^{2}}{24} \cdot \frac{(p-1)^{2} \cdot(p-5)}{p^{2}} .
$$

If $p \equiv 1 \bmod 4$, then taking $c=4$ in (6), from (4) we can deduce the identity

$$
\sum_{\substack{\chi \bmod p \\ \chi(-1)=-1}} \chi(4) \cdot|L(1, \chi)|^{2}=\frac{\pi^{2}}{48} \cdot \frac{(p-1)^{2} \cdot(p-17)}{p^{2}} .
$$

If $p \equiv 3 \bmod 4$, then from (4) and (7) we have the identity

$$
\sum_{\substack{\chi \bmod p \\ \chi(-1)=-1}} \chi(4) \cdot|L(1, \chi)|^{2}=\frac{\pi^{2}}{48} \cdot \frac{(p-1) \cdot\left(p^{2}-6 p+17\right)}{p^{2}} .
$$

Now Lemma 5 follows from (8)-(11).

\section{Proof of the theorems}

In this section, we shall complete the proof of our theorems. Note that if $\chi$ is a nonprincipal character $\bmod p$, then $|\tau(\chi)|=\sqrt{p}$ and

$$
\left|\sum_{m=1}^{p-1} \chi(m) K(m, p)\right|=\left|\sum_{a=1}^{p-1} \sum_{m=1}^{p-1} \chi(m) e\left(\frac{m a+\bar{a}}{p}\right)\right|=\left|\tau^{2}(\chi)\right|=p .
$$

If $p \equiv 3 \bmod 4$, then from (12), Lemma 4 and Lemma 5 we have

$$
\begin{aligned}
& \sum_{m=1}^{p-1} \sum_{n=1}^{p-1} K(m, p) \cdot K(n, p) \cdot S_{1}(2 m \cdot \bar{n}, p) \\
&=-\frac{20 \cdot p}{\pi^{2}(p-1)} \sum_{\chi \bmod p} \chi(2)\left|\sum_{n=1}^{p-1} \chi(n) \cdot K(n, p)\right|^{2} \cdot|L(1, \chi)|^{2} \\
&+\frac{8 \cdot p}{\pi^{2}(p-1)} \sum_{\chi \bmod p} \chi(4)\left|\sum_{n=1}^{p-1} \chi(n) \cdot K(n, p)\right|^{2} \cdot|L(1, \chi)|^{2} \\
&+\frac{8 \cdot p}{\pi^{2}(p-1)=-1} \sum_{\chi \bmod p}\left|\sum_{n=1}^{p-1} \chi(n) \cdot K(n, p)\right|^{2} \cdot|L(1, \chi)|^{2} \\
&=-\frac{20 \cdot p^{3}}{\pi^{2}(p-1)=-1} \sum_{\chi \bmod p} \chi(2) \cdot|L(1, \chi)|^{2}+\frac{8 \cdot p^{3}}{\pi^{2}(p-1)} \sum_{\chi \bmod p}|L(1, \chi)|^{2} \\
& \chi(-1)=-1 \\
&+\frac{8 \cdot p^{3}}{\pi^{2}(p-1)} \sum_{\chi \bmod p} \chi(4) \cdot|L(1, \chi)|^{2} \\
& \chi(-1)=-1 \\
&=-\frac{5}{6} p(p-1)(p-5)+\frac{2}{3} p(p-1)(p-2)+\frac{1}{6} p\left(p^{2}-6 p+17\right)=2 p^{2} .
\end{aligned}
$$


If $p \equiv 1 \bmod 4$, then from (12), Lemma 4 and Lemma 5 we also have

$$
\begin{aligned}
& \sum_{m=1}^{p-1} \sum_{n=1}^{p-1} K(m, p) \cdot K(n, p) \cdot S_{1}(2 m \cdot \bar{n}, p) \\
& =-\frac{20 \cdot p^{3}}{\pi^{2}(p-1)} \sum_{\substack{\chi \bmod p \\
\chi(-1)=-1}} \chi(2) \cdot|L(1, \chi)|^{2}+\frac{8 \cdot p^{3}}{\pi^{2}(p-1)} \sum_{\substack{\chi \bmod p \\
\chi(-1)=-1}}|L(1, \chi)|^{2} \\
& \quad+\frac{8 \cdot p^{3}}{\pi^{2}(p-1)} \sum_{\substack{\chi \bmod p \\
\chi(-1)=-1}} \chi(4) \cdot|L(1, \chi)|^{2} \\
& =-\frac{5}{6} p(p-1)(p-5)+\frac{2}{3} p(p-1)(p-2)+\frac{1}{6} p(p-1)(p-17)=0 .
\end{aligned}
$$

It is clear that Theorem 1 follows from (13) and (14).

Now we prove Theorem 2 . If $p \equiv 1 \bmod 4$, then from Lemma 1 and the method of proving Theorem 1 we have

$$
\begin{aligned}
& \sum_{m=1}^{p-1} \sum_{n=1}^{p-1}|K(m, p)|^{2} \cdot|K(n, p)|^{2} \cdot S_{1}(2 m \cdot \bar{n}, p) \\
& =-\left.\left.\frac{20 \cdot p}{\pi^{2}(p-1)} \sum_{\substack{\chi \bmod p \\
\chi(-1)=-1}} \chi(2)\left|\sum_{n=1}^{p-1} \chi(n) \cdot\right| K(n, p)\right|^{2}\right|^{2} \cdot|L(1, \chi)|^{2} \\
& +\left.\left.\frac{8 \cdot p}{\pi^{2}(p-1)} \sum_{\substack{\chi \bmod p \\
\chi(-1)=-1}} \chi(4)\left|\sum_{n=1}^{p-1} \chi(n) \cdot\right| K(n, p)\right|^{2}\right|^{2} \cdot|L(1, \chi)|^{2} \\
& +\left.\left.\frac{8 \cdot p}{\pi^{2}(p-1)} \sum_{\substack{\chi \bmod p \\
\chi(-1)=-1}}\left|\sum_{n=1}^{p-1} \chi(n) \cdot\right| K(n, p)\right|^{2}\right|^{2} \cdot|L(1, \chi)|^{2} \\
& =-\frac{20 \cdot p^{4}}{\pi^{2}(p-1)} \sum_{\substack{\chi \bmod p \\
\chi(-1)=-1}} \chi(2) \cdot|L(1, \chi)|^{2}+\frac{8 \cdot p^{4}}{\pi^{2}(p-1)} \sum_{\substack{\chi \bmod p \\
\chi(-1)=-1}}|L(1, \chi)|^{2} \\
& +\frac{8 \cdot p^{4}}{\pi^{2}(p-1)} \sum_{\substack{\chi \bmod p \\
\chi(-1)=-1}} \chi(4) \cdot|L(1, \chi)|^{2} \\
& =-\frac{5}{6} p^{2}(p-1)(p-5)+\frac{2}{3} p^{2}(p-1)(p-2)+\frac{1}{6} p^{2}(p-1)(p-17)=0 \text {. }
\end{aligned}
$$

If $p \equiv 3 \bmod 4$, then note that the Legendre symbol $\left(\frac{-1}{p}\right)=\chi_{2}(-1)=-1, L\left(1, \chi_{2}\right)=\pi \cdot h_{p} / \sqrt{p}$, and

$$
\sum_{a=1}^{p-1}\left(\frac{a}{p}\right)^{2} e\left(\frac{a}{p}\right)=-1
$$


so from Lemma 1 and the method of proving Theorem 1 we have

$$
\begin{aligned}
& \sum_{m=1}^{p-1} \sum_{n=1}^{p-1}|K(m, p)|^{2} \cdot|K(n, p)|^{2} \cdot S_{1}(2 m \cdot \bar{n}, p) \\
& =-\left.\left.\frac{20 \cdot p}{\pi^{2}(p-1)} \sum_{\substack{\chi \bmod p \\
\chi(-1)=-1}} \chi(2)\left|\sum_{n=1}^{p-1} \chi(n) \cdot\right| K(n, p)\right|^{2}\right|^{2} \cdot|L(1, \chi)|^{2} \\
& +\left.\left.\frac{8 \cdot p}{\pi^{2}(p-1)} \sum_{\substack{\chi \bmod p \\
\chi(-1)=-1}} \chi(4)\left|\sum_{n=1}^{p-1} \chi(n) \cdot\right| K(n, p)\right|^{2}\right|^{2} \cdot|L(1, \chi)|^{2} \\
& +\left.\left.\frac{8 \cdot p}{\pi^{2}(p-1)} \sum_{\substack{\chi \bmod p \\
\chi(-1)=-1}}\left|\sum_{n=1}^{p-1} \chi(n) \cdot\right| K(n, p)\right|^{2}\right|^{2} \cdot|L(1, \chi)|^{2} \\
& =-\frac{20 \cdot p^{4}}{\pi^{2}(p-1)} \sum_{\substack{\chi \bmod p \\
\chi(-1)=-1}} \chi(2) \cdot|L(1, \chi)|^{2}+\frac{8 \cdot p^{4}}{\pi^{2}(p-1)} \sum_{\substack{\chi \bmod p \\
\chi(-1)=-1}}|L(1, \chi)|^{2} \\
& +\frac{8 \cdot p^{4}}{\pi^{2}(p-1)} \sum_{\substack{\chi \bmod p \\
\chi(-1)=-1}} \chi(4) \cdot|L(1, \chi)|^{2}+\frac{20 \cdot p^{3}}{\pi^{2}} \cdot\left(\frac{2}{p}\right) \cdot\left|L\left(1, \chi_{2}\right)\right|^{2} \\
& -\frac{8 \cdot p^{3}}{\pi^{2}} \cdot\left(\frac{4}{p}\right) \cdot\left|L\left(1, \chi_{2}\right)\right|^{2}-\frac{8 \cdot p^{3}}{\pi^{2}} \cdot\left|L\left(1, \chi_{2}\right)\right|^{2} \\
& =-\frac{5}{6} p^{2}(p-1)(p-5)+\frac{2}{3} p^{2}(p-1)(p-2)+\frac{1}{6} p^{2}\left(p^{2}-6 p+17\right) \\
& +20 \cdot p^{2} \cdot h_{p}^{2} \cdot\left(\frac{2}{p}\right)-16 \cdot p^{2} \cdot h_{p}^{2} \\
& =2 p^{3}+20 \cdot\left(\frac{2}{p}\right) \cdot p^{2} \cdot h_{p}^{2}-16 \cdot p^{2} \cdot h_{p}^{2} .
\end{aligned}
$$

Note that $\left(\frac{2}{p}\right)=(-1)^{\frac{p^{2}-1}{8}}=-1$ if $p \equiv 3 \bmod 8$, and $\left(\frac{2}{p}\right)=1$ if $p \equiv 7 \bmod 8$, then from (15) and (16) we may immediately deduce Theorem 2 . This completes the proof of our theorems.

\section{Competing interests}

The authors declare that they have no competing interests.

\section{Authors' contributions}

$\mathrm{HZ}$ obtained the theorems and completed the proof. WZ corrected and improved the final version. Both authors read and approved the final manuscript.

\section{Acknowledgements}

The authors express their gratitude to the referee for very helpful and detailed comments. This work is supported by the N.S.F. (11371291), the S.R.F.D.P. (20136101110014), the N.S.F. (2013JZ001) of Shaanxi Province, P.R. China.

Received: 12 December 2013 Accepted: 13 January 2014 Published: 31 Jan 2014

\section{References}

1. Rademacher, $\mathrm{H}$ : On the transformation of $\log \eta(\tau)$. J. Indian Math. Soc. 19, 25-30 (1955)

2. Rademacher, H: Dedekind Sums. Carus Mathematical Monographs. Math. Assoc. of America, Washington (1972)

3. Berndt, BC: Analytic Eisenstein series, theta-functions, and series relations in the spirit of Ramanujan. J. Reine Angew. Math. 303/304, 332-365 (1978)

4. Apostol, TM: Modular Functions and Dirichlet Series in Number Theory. Springer, New York (1976) 
5. Carlitz, L: The reciprocity theorem of Dedekind sums. Pac. J. Math. 3, 513-522 (1953)

6. Conrey, JB, Fransen, E, Klein, R, Clayton, S: Mean values of Dedekind sums. J. Number Theory 56, $214-226$ (1996)

7. Sitaramachandraro, R: Dedekind and Hardy sums. Acta Arith. 48, 325-340 (1987)

8. Zhang, W, Yi, Y: On the 2m-th power mean of certain Hardy sums. Soochow J. Math. 26, 73-84 (2000)

9. Zhang, W: On the mean values of Dedekind sums. J. Théor. Nr. Bordx. 8, 429-442 (1996)

10. Chowla, S: On Kloosterman's sum. Nor. Vidensk. Selsk. Fak. Frondheim 40, 70-72 (1967)

11. Malyshev, AV: A generalization of Kloosterman sums and their estimates. Vestn. Leningr. Univ. 15, 59-75 (1960) (in Russian)

12. Apostol, TM: Introduction to Analytic Number Theory. Springer, New York (1976)

10.1186/1029-242X-2014-52

Cite this article as: Zhang and Zhang: On the identity involving certain Hardy sums and Kloosterman sums. Journal of Inequalities and Applications 2014, 2014:52

\section{Submit your manuscript to a SpringerOpen ${ }^{\circ}$ journal and benefit from:}

- Convenient online submission

- Rigorous peer review

- Immediate publication on acceptance

- Open access: articles freely available online

- High visibility within the field

- Retaining the copyright to your article 